\title{
Reservoir water level forecasting using normalization and multiple regression
}

\author{
Siti Rafidah M-Dawam ${ }^{1}$, Ku Ruhana Ku-Mahamud ${ }^{2}$ \\ ${ }^{1}$ Faculty of Computer and Mathematical Sciences, Universiti Teknologi MARA Kedah, Malaysia \\ ${ }^{2}$ School of Computing, College of Arts and Sciences, Universiti Utara Malaysia, Sintok, Kedah, Malaysia
}

\begin{tabular}{l}
\hline Article Info \\
\hline Article history: \\
Received Oct 6, 2018 \\
Revised Nov 19, 2018 \\
Accepted Dec 27, 2018 \\
\hline
\end{tabular}

Keywords:

Forecasting model Reservoir modelling

Reservoir water release

Sliding window

Temporal data mining

\begin{abstract}
Many non-parametric techniques such as Neural Network (NN) are used to forecast current reservoir water level (RWLt). However, modelling using these techniques can be established without knowledge of the mathematical relationship between the inputs and the corresponding outputs. Another important issue to be considered which is related to forecasting is the preprocessing stage where most non-parametric techniques normalize data into discretized data. Data normalization can influence the the results of forecasting. This paper presents reservoir water level (RWL) forecasting using normalization and multiple regression. In this study, continuous data of rainfall (RF) and changes of reservoir water level (WC) are normalized using two different normalization methods, Min-Max and Z-Score techniques. Its comparative studies and forecasting process are carried out using multiple regression. Three input scenarios for multiple regression were designed which comprise of temporal patterns of $\mathrm{WC}$ and $\mathrm{RF}$, in which the sliding window technique has been applied. The experimental results showed that the best input scenario for forecasting the RWLt employs both the RF and the $\mathrm{WC}$, in which the best predictors are three day's delay of WC and two days' delay of RF. The findings also suggested that the performance of the RWL forecasting model using multiple regression was dependent on the normalization methods.
\end{abstract}

Copyright (ㅇ 2019 Institute of Advanced Engineering and Science. All rights reserved.

\section{Corresponding Author:}

Siti Rafidah M-Dawam,

Faculty of Computer and Mathematical Sciences,

Universiti Teknologi MARA Kedah,

P.O. Box 187, 08400 Merbok, Kedah, Malaysia.

Email: srafidah192@kedah.uitm.edu.my

\section{INTRODUCTION}

Forecasting RWL is crucial for reservoir's operator in making decision on the reservoir water release (RWR) of a particular reservoir. It is a challenging and complex task, especially during flood and drought occurances due to unpredictable inflow such as RF [1]. Thus, a few researches have focused on nonstructural approaches predicting reservoir inflows [2]. However, during flood or drought, the decision on RWR is not only based on the availability of water inflows, but also on the previous release, demands, time, etc. Besides daily RF, several researches also considered changes in the RWL (WC) as an input in the multipurpose reservoir forecasting model [2]. RF (hydrological data) and reservoir WC are found to be correlated in the flood prediction model [3].

Many literature conducted on the RWR operation have utilized RF data and RWL as inputs [4], and have applied different methods and techniques of Artificial Intelligence and machine learning[5-8]. Only a small number of researches conducted on RWR decisions highlighted on the time delay between the RF and the increase of RWL. 
In [9] discretized data are normalized using Min-Max technique. In this study, the results showed eight days' time lag relating to upstream RF and RWL with an ANN model of 24-15-3. Later, the model recommended five days' time lag with 8-23-2 ANN model with a $0.007085 \%$ error. Type 2 SVM regression has been used by [2] to forecast the daily RWL of the Klang reservoir, Malaysia. The study employed ZScore technique for data normalization and found out that the best input variables are combination of both RF and RWL, which were used to determine the best time lag which are two days of RF and with $1.64 \%$ error. Autoregressive Integrated Moving Average (ARIMA) model was developed in [4] for predicting the Kainji Dam, Nigeria daily water levels using a ten-year record. The study resulted in a model with a relative error of $0.039 \%$ had the best prediction. In [10] ANN with feedforward back propagation was concluded as the suitable predictor for real-time water level forecasting of the Sukhi Reservoir, India. The inputs are the daily data of inflow, RWL, and RWR where the best time lag is ten days with a $0.82 \%$ error. NN was also employed in [11] to predict RWL and concluded a 5-25-1 NN model as the best architecture. The study found out that five days' observations of RWL are significant for the RWR decision with a $0.038756 \%$ error. A NN architecture of 4-17-1 in forecasting the change of RWL stage was proposed in [3]. The input patterns were the changes and stages of RWL instead of the real value of RWL. The research showed that the changes in the stages of RWL were influenced by the two days of delay. However, modelling using NN techniques can be established without knowledge of the mathematical relationship between the inputs and the corresponding outputs. Whereas multiple regression is used to explore the relationship between one continuous dependent variable (DV) and a number of independent variables (IVs) or predictors (usually continuous). It can determine how well a set of variables is able to predict a particular outcome [12-18]. This study applied multiple regression in order to identify which IVs (slices of RWL and RF) can best be the input predictors to predict DV $\left(\mathrm{RWL}_{\mathrm{t}}\right)$.

Another important issue to be considered which is related to forecasting is during the preprocessing phase where most non-parametric techniques normalize data into discretized data. Data normalization can influence the results of forecasting. Normalization can be performed at the level of the input features or at the level of the kernel [19]. In many applications, the available features are continuous values, where each feature is measured in a different scale and has a different range of possible values. In such cases, it is often beneficial to scale all features to a common range by standardizing the data. Previous studies mentioned above, have not reported any comparative study done on the normalization method used in their research. In [19-22], normalization process has increased the classification accuracy while in certain datasets, normalization may not demonstrate significant advantages [23] .

In RWL forecasting, the data is in the form of temporal sequences, where time (month, day or hours) is critical [24]. The changes in the patterns of the data can influence certain decision-making. The Temporal Data Mining (TDM) technique is required to uncover the values of the attributes involved from temporal sequences representing temporal information related to certain decisions by the algorithm formulation. The significant time delay between the cause of event and the actual event needs to be captured accurately. Several studies reported on the use of temporal data in forecasting [3], [11], [25-33].

This paper presents reservoir water level (RWL) forecasting using normalization and multiple regression. In this study, continuous data of RF and changes of reservoir water level (WC) are normalized using two different normalization methods, Min-Max and Z-Score techniques. Its comparative studies and forecasting process are carried out using multiple regression. Three input scenarios for multiple regression were designed which comprise of temporal patterns of WC and RF. The sliding window technique has been used to capture the delay in temporal data. The experimental results showed that the best input scenario for forecasting the $\mathrm{RWL}_{\mathrm{t}}$ employs both the RF and the WC, in which the best predictors are three day's delay of WC and two days' delay of RF. The findings also suggested that the performance of the RWL forecasting model using multiple regression was dependent on the normalization methods. Root Mean Square (RMSE), Mean Absolute Error (MAE) and Mean Absolute Percentage Error (MAPE) have been used as the parameters to measure the forecast results based on the actual data analysis.

\section{RESEARCH METHOD}

Figure 1 depicts the approach that has been used in conducting the research. The reservoir data which consist of RF and RWL from 1997 until 2006, have been collected from the Department of Irrigation and Drainage (DID), which is in charge of monitoring and managing the Timah Tasoh reservoir. This reservoir is one of the largest multipurpose reservoirs situated in the northern Peninsular of Malaysia.The data consists of operational and hydrological data. The operational data has the daily RWLs measured in metre $(\mathrm{m})$ unit while the hydrological data has the daily RF readings measured in milimetre $(\mathrm{mm})$, recorded from five gauging stations.

Indonesian J Elec Eng \& Comp Sci, Vol. 14, No. 1, April 2019 : 443 - 449 


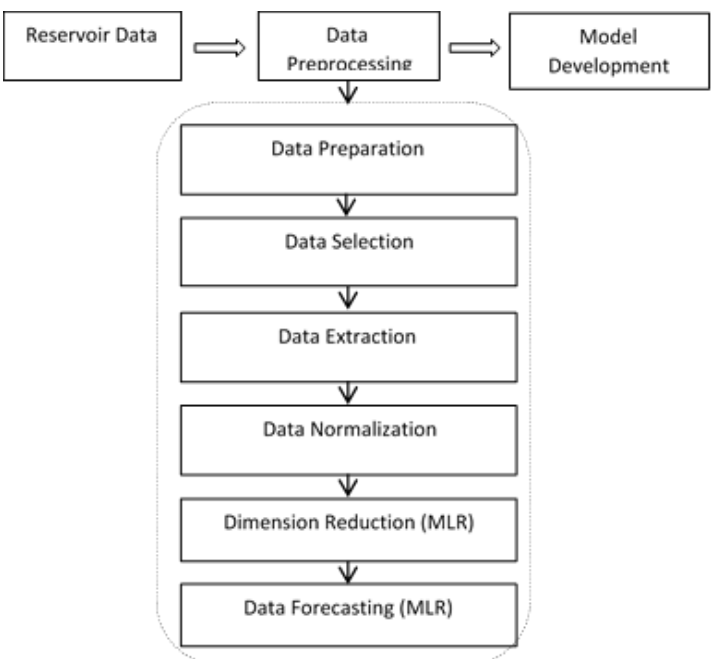

Figure 1. The process flow for RWL forecasting

In the data preparation stage, the attributes are described and records with missing values were interpolated. This study used the RWL as the output whiles the changes of the reservoir water level (WC) and $\mathrm{RF}$ were used as the input. These WC will be calculated using equation [3](1):

$$
W C_{t}=R W L_{t}-R W L_{t-1}
$$

where $\mathrm{WC}_{\mathrm{t}}$ is the change of RWL at current time $t, \mathrm{RWL}_{\mathrm{t}}$ is the RWL at current time $t$ and $\mathrm{RWL}_{\mathrm{t}-1}$ is the $\mathrm{RWL}$ at one previous day $t-1$. The RF data are averaged by the number of stations that have RF based on [30] (2):

$$
\text { Average_RF }=\frac{\text { total_rain }}{\text { number_of_stations_with_rain }}
$$

Next, the change-point detection technique is applied, where records which consist of gate opening decision only are extracted [34] while records with gate closing decision were removed. A total of 501 records were detected from ten years of reservoir operation (1997-2006).

The RF and WC data used in this study is temporal data with the time delayed event. The changes in RWL are the impact of several sequences events of RF. In order to capture the temporal information of WC and RF, sliding window technique is applied [34]. Figure 2 shows the pseudo-code for the sliding window where $n$ is the size of the window. In this study, $n$ is taken as the value of seven to investigate on the effect of seven previous event on current RWL [35] as showed in Table 1 and Table 2.

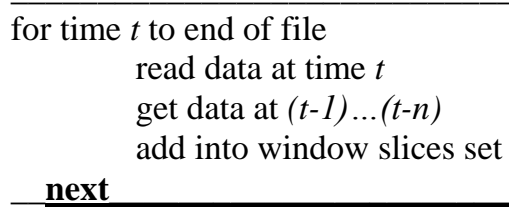

Figure 2. Steps for Sliding Window

Table 1. Sliced Reservoir WC

\begin{tabular}{ccccccccc}
\hline Date & RWLt & WCt-1 & WCt-2 & WCt-3 & WCt-4 & WCt-5 & WCt-6 & WCt-7 \\
\hline 12-Feb-97 & 29.275 & 0.020 & 0.035 & 0.055 & 0.035 & 0.025 & 0.150 & 0.005 \\
13-Feb-97 & 29.335 & 0.060 & 0.020 & 0.035 & 0.055 & 0.035 & 0.025 & 0.150 \\
14-Feb-97 & 29.335 & 0.000 & 0.060 & 0.020 & 0.035 & 0.055 & 0.035 & 0.025 \\
. &. &. &. &. &. &. &. &. \\
. &. &. &. &. &. &. &. &. \\
\hline
\end{tabular}




\begin{tabular}{cccccrrrr}
\multicolumn{10}{c}{ Table 2. Sliced RF } \\
\hline Date & Average_RF & RFt-1 & RFt-2 & RFt-3 & RFt-4 & RFt-5 & RFt-6 & RFt-7 \\
\hline 12-Feb-97 & 20.250 & 7.330 & 5.380 & 13.00 & 0.000 & 46.250 & 24.500 \\
13-Feb-97 & 13.875 & 20.250 & 7.330 & 5.380 & 13.000 & 0.000 & 46.250 & 24.000 \\
14-Feb-97 & 8.250 & 13.880 & 20.250 & 7.330 & 5.380 & 13.000 & 0.000 & 46.250 \\
. &. &. &. &. &. &. &. \\
. &. &. &. &. &. &. &. \\
\hline
\end{tabular}

In the next stage, the reservoir WC and RF are normalized, where the attribute data is scaled so as to fall within a small specified range. In a real application, because of the differences in the range of attributes' values, one attribute might overpower the other. Normalization prevents the outweighing attributes with a large range. The goal is to equalize the size or magnitude and the variability of these attributes. There are many types of data normalization, however only two techniques are used to make a comparison in this study; Z-Score and Min-Max Normalization.

In Z-Score normalization, the values for the attributes of reservoir WC and RF are normalized based on the mean and standard deviation. The equation for such transformation is given as follows (3):

$$
Z_{\text {new }}=\frac{Z-\bar{Z}}{S D}
$$

where $\bar{Z}$ is the mean of attribute and $S D$ is the standard deviation of the attribute. This method of normalization is useful if the actual minimum and maximum values of the attributes are unknown. The advantage of this statistical norm is that it reduces the effects of outliers in the data. Table 3 and Table 4 showed the normalized WC and RF using Z-Score technique.

Table 3. Z-Score of Reservoir WC

\begin{tabular}{ccccccccc}
\hline Date & zRWLt & zWCt-1 & zWCt-2 & zWCt-3 & zWCt-4 & zWCt-5 & zWCt-6 & zWCt-7 \\
\hline 12-Feb-97 & 0.694 & 0.266 & 0.292 & 0.393 & 0.148 & 0.017 & 1.310 & -0.204 \\
13-Feb-97 & 0.908 & 0.627 & 0.156 & 0.207 & 0.337 & 0.116 & 0.003 & 1.349 \\
14-Feb-97 & 0.908 & 0.086 & 0.519 & 0.067 & 0.148 & 0.314 & 0.108 & 0.010 \\
. &. &. &. &. &. &. &. &. \\
. &. &. &. &. &. &. &. &. \\
\hline
\end{tabular}

Table 4. Z-Score of RF

\begin{tabular}{ccccccrrr}
\hline Date & zRFt & zRFt-1 & zRFt-2 & zRFt-3 & zRFt-4 & zRFt-5 & zRFt-6 & zRFt-7 \\
\hline 12-Feb-97 & 0.433 & -0.463 & -0.617 & -0.192 & -1.039 & 1.938 & 0.556 & -0.351 \\
13-Feb-97 & 0.022 & 0.298 & -0.503 & -0.642 & -0.191 & -1.038 & 1.979 & 0.605 \\
14-Feb-97 & -0.340 & -0.077 & 0.254 & -0.527 & -0.688 & -0.201 & -1.045 & 2.049 \\
. &. &. &. &. &. &. &. &. \\
. &. &. &. &. &. &. &. & \\
\hline
\end{tabular}

The second technique is Min-Max Normalization. This method rescales the attributes or outputs from one range of values to a new range of values. The attributes are rescaled to lie within a range of 0 to 1 or from -1 to 1 . The rescaling is accomplished by using the following equation (4):

$$
M_{\text {new }}=\frac{M-M_{\min }}{M_{\max }-M_{\min }}
$$

where $M$ is the actual value of an attribute. This method has the advantage of preserving exactly all relationships in the data. Table 5 and Table 6 showed the normalized WC and RF using Min-Max technique.

Table 5. Min-Max of Reservoir WC

\begin{tabular}{cccccrrrr}
\hline Date & mRWLt & mWCt-1 & mWCt-2 & mWCt-3 & mWCt-4 & mWCt-5 & mWCt-6 & mWCt-7 \\
\hline 12-Feb-97 & 0.5838 & 0.2735 & 0.2863 & 0.3034 & 0.2947 & 0.2863 & 0.3918 \\
13-Feb-97 & 0.6185 & 0.3076 & 0.2735 & 0.2863 & 0.3116 & 0.2947 & 0.2863 \\
14-Feb-97 & 0.6185 & 0.2564 & 0.3076 & 0.2735 & 0.2947 & 0.3116 & 0.2947 \\
. &. &. &. &. &. &. &. &. \\
. &. &. &. &. &. &. &. \\
\hline
\end{tabular}




\begin{tabular}{ccccccccc}
\multicolumn{10}{c}{ Table 6. Min-Max of RF } \\
\hline Date & mRFt & mRFt-1 & mRFt-2 & mRFt-3 & mRFt-4 & mRFt-5 & mRFt-6 & mRFt-7 \\
\hline 12-Feb-97 & 0.1387 & 0.0502 & 0.0368 & 0.0890 & 0.0000 & 0.3846 & 0.2037 & 0.0831 \\
13-Feb-97 & 0.0950 & 0.1387 & 0.0502 & 0.0368 & 0.1081 & 0.0000 & 0.3846 & 0.2037 \\
14-Feb-97 & 0.0565 & 0.0951 & 0.1387 & 0.0502 & 0.0447 & 0.1081 & 0.0000 & 0.3846 \\
. &. &. &. &. &. &. &. &. \\
\hline &. &. &. &. &. &. &. \\
\hline
\end{tabular}

Multiple regression is used to explore the relationship between one continuous dependent variable (DV) and a number of independent variables (IVs) or predictors (usually continuous). It can determine how well a set of variables is able to predict a particular outcome. The regression equation (5) takes the following form:

$$
Y=A+B_{1} X_{1}+B_{2} X_{2}+\ldots .+B_{n} X_{n}
$$

where $Y^{`}$ is the predicted value on the $\mathrm{DV}, A$ is the intercept, the $X s$ represent the various IVs, and the $B s$ are the coefficients assigned to each of the IVs during regression.

The ouput for this study is the $\mathrm{RWL}_{\mathrm{t}}$ and the inputs are reservoir WC and RF. This study designed three different input scenarios for multiple regression in order to identify which input scenarios (IVs) can best be the input predictors to forecast $\mathrm{RWL}_{\mathrm{t}}(\mathrm{DV})$. The first scenario considers the daily RF between time $(t-1)$ and $(t-7)$ as the sole input, while the second scenario considers both the RF (at $t-1-t-7$ ) dan reservoir WC (at $t-1-t-7)$ as inputs. The third scenario uses the reservoir WC only between time $(t-1)$ and $(t-7)$ as inputs. Equations (6), (7) and (8) represent the first, second and third scenarios, respectively.

$$
\begin{aligned}
& \mathrm{RWL}_{\mathrm{t}}=f \mathrm{RF}(\mathrm{t}-i) \quad i=\{-1,-2,-3,-4,-5,-6,-7\} \\
& \mathrm{RWL}_{\mathrm{t}}=f(\mathrm{RF}(\mathrm{t}-i), \mathrm{WC}(\mathrm{t}-j)) i=\{-1,-2,-3,-4,-5,-6,-7\} \quad j=\{-1,-2,-3,-4,-5,-6,-7\} \\
& \mathrm{RWL}_{\mathrm{t}}=f \mathrm{WC}(\mathrm{t}-i) \quad i=\{-1,-2,-3,-4,-5,-6,-7\}
\end{aligned}
$$

\section{RESULTS AND ANALYSIS}

In this section, the results of the study are discussed based on inputs scenario and data normalization technique.The best input scenario is determined before proceeding further into the forecasting calculation. Based on statistical test in Table 7, the forecasted values obtained by employing second input scenario achieve the best results from other two scenarios. The scenario employs more input data, thus providing a better forecasting estimation. It has greater $\mathrm{R}^{2}$ which is 0.319 as compared to the first and second scenario which has $\mathrm{R}^{2}$ values equal to 0.193 and 0.279 respectively. The second input scenario also has smaller standard error of estimate (SEE) for both normalization methods. The SEE for Min-Max Technique is 0.13588 , and SEE for Z-Score technique is 0.833856 . Therefore, this second input scenario will be used as the best inputs for further data runs.

Table 7. Statistical Test for Three Input Scenarios

\begin{tabular}{lcccc}
\hline Input Scenario & $\mathrm{R}$ & $\mathrm{R}^{2}$ & SEE (Min-Max Technique) & SEE (Z-Score Technique) \\
\hline First & 0.440 & 0.193 & 0.14673 & 0.90548 \\
Second & 0.565 & $\mathbf{0 . 3 1 9}$ & $\mathbf{0 . 1 3 5 8 8}$ & $\mathbf{0 . 8 3 8 5 6}$ \\
Third & 0.528 & 0.279 & 0.13872 & 0.85607 \\
\hline
\end{tabular}

The sliding window technique has been successfully applied on RWL data to extract and segment the temporal data and preserved the delay. The study used multiple regression to find out that the best time lag for forecasting $\mathrm{RWL}_{\mathrm{t}}$ is three days' delay of reservoir WC and two days of RF. Based on this finding, two set of regression model for $\mathrm{RWL}_{t}$ are developed in order to investigate which normalization techniques produces less error. The first regression model used the Min-Max while the second model used Z-Score normalization technique as shown in equation (9) and (10):

$$
\begin{aligned}
& \mathrm{RWL}_{\mathrm{t}}=(0.175)+(0.375) \mathrm{mWC}_{\mathrm{t}-2}+(0.228) \mathrm{mWC}_{\mathrm{t}-3}+(0.358) \mathrm{mWC}_{\mathrm{t}-4}+(0.172) \mathrm{mRF}_{\mathrm{t}-1}+(0.183) \mathrm{mRF}_{\mathrm{t}-2} \\
& \mathrm{RWL}_{\mathrm{t}}=(0.00)+(0.218) \mathrm{zWC}_{\mathrm{t}-2}+(0.129) \mathrm{zWC}_{\mathrm{t}-3}+(0.197) \mathrm{zWC}_{\mathrm{t}-4}+(0.123) \mathrm{zRF}_{\mathrm{t}-1}+(0.132) \mathrm{zRF}_{\mathrm{t}-2}
\end{aligned}
$$


Two sets of data based on two different data normalization were tested using the two regression model developed. Four statistical formula are selected to evaluate the forecasting efficiency in this study, namely Root Mean Square (RMSE), Mean Absolute Error (MAE), Mean Absolute Percentage Error (MAPE) and the Correlation Coefficient (R). The comparison of statistical evaluation on two normalization techniques is shown in Table 8. The results showed that the obtained values of RMSE, MAPE and MAE by using Min-Max technique are $0.14125,0.24191$ and 0.11122 respectively. While using the Z-Score technique the results are $0.87165,6.90884$ and 0.68677 respectively. All the RMSE, MAE and MAPE values obtained using Min-Max data normalization are closer to 0 than using Z-Score technique, indicating that the Min-Max techniques is better than Z-Score. However, the Z-Score technique provides slightly greater correlation coefficient values $(\mathrm{R}=0.48858)$, than the Min-Max technique $(\mathrm{R}=0.48856)$. In overall, forecasting using Min-Max data normalization techniques yield less error than using the Z-Score technique. The predicted output using MinMax normalization is more reliable than that of the Z-Score normalization technique.

Table 8. Comparison of Statistical Evaluation for Normalization Technique

\begin{tabular}{ccccc}
\hline Normalization Technique & RMSE & MAPE & MAE & R \\
\hline Min-Max & 0.14125 & 0.24191 & 0.11122 & 0.48856 \\
Z-Score & 0.87165 & 6.90884 & 0.68677 & 0.48858 \\
\hline
\end{tabular}

\section{CONCLUSION}

This paper has presented reservoir water level (RWL) forecasting using normalization and multiple regression. The research on the comparison of input scenario for multiple regression concludes that the best input scenario for multiple regression is the second input scenario which consists of combination data of RF and WC.

The sliding window technique has been successfully applied on RWL data to extract and segment the temporal data and preserved the delay. The study used multiple regression to find out that the best time lag for forecasting $\mathrm{RWL}_{\mathrm{t}}$ is three days' delay of reservoir WC and two days of RF.

The comparative studies on the two different normalization methods of the Timah Tasoh reservoir data using multiple regression showed that data normalized using Min-Max technique can enhance the reliability of the forecasting model for $\mathrm{RWL}_{\mathrm{t}}$. Forecasting using Min-Max techniques yield less error than using the Z-Score technique and the predicted output is more reliable. The experimental results showed that the prediction of the RWL $\mathrm{R}_{\mathrm{t}}$ using MLR was dependent on the normalization methods used.

In the future, other input variables such as sediment, volume of water release and spatial effect can be explored to improve the forecasting model of $\mathrm{RWL}_{\mathrm{t}}$. The comparison of other various statistical normalization methods such as median, sigmoid and statistical column normalization can also be measured.

\section{ACKNOWLEDGEMENTS}

The authors wish to thank the Ministry of Higher Education Malaysia for funding this study under the Long Term Research Grant Scheme (LRGS/b-u/2012/UUM/Teknologi Komunikasi dan Informasi), and the Department of Irrigation and Drainage Malaysia for supplying hydrology and reservoir operational data.

\section{REFERENCES}

[1] T. M. Sattari, K. Yurekli, and M. Pal, "Performance evaluation of artificial neural network approaches in forecasting reservoir inflow," Appl. Math. Model., vol. 36, no. 6, pp. 2649-2657, Jun. 2012.

[2] H. Afiq, E. Ahmed, N. Ali, K. Othman Abdul, H. Aini, and M. Muhammad, "Daily Forecasting of Dam Water Levels: Comparing a Support Vector Machine (SVM) Model With Adaptive Neuro Fuzzy Inference System (ANFIS)," Water Resour. Manag., vol. 27, no. 10, pp. 3803-3823, Jun. 2013.

[3] N. Ashaary, W. IShak, and K. Ku-Mahamud, "Neural Network Application in the change of reservoir water level stage forecasting," Indian J. Sci. Technol., vol. 8, no. 13, pp. 1-6, 2015.

[4] C. C. Nwobi-Okoye and A. C. Igboanugo, "Predicting Water Levels at Kainji Dam using Artificial Neural Networks," Niger. J. Technol., vol. 32, no. 1, pp. 129-136, 2013.

[5] A. Afshar and A. Salehi, "Gated Spillways Operation Rules Considering Water Surface Elevation and Flood Peak; Application to Karkheh Dam,” World Environ. Water Resour. Congr. 2011, no. 2000, pp. 3007-3015, 2011.

[6] M. H. Afshar, "Large scale reservoir operation by Constrained Particle Swarm Optimization algorithms," J. Hydroenvironment Res., vol. 6, no. 1, pp. 75-87, Mar. 2012.

[7] E. T. Alemu, R. N. Palmer, A. Polebitski, and B. Meaker, "Decision Support System for Optimizing Reservoir Operations Using Ensemble Streamflow Predictions," J. Water Resour. Plan. Manag., vol. 137, no. February, pp. 72-82, 2011. 
[8] C.-C. Wei, "Discretized and Continuous Target Fields for the Reservoir Release Rules During Floods," Water Resour. Manag., vol. 26, no. 12, pp. 3457-3477, Jun. 2012.

[9] W. H. Ishak, K. R. Ku-Mahamud, and N. Norwawi, "Neural Network Application in Reservoir Water Level Forecasting and Release Decision,” Int. J. New Comput. Archit. Their Appl., vol. 1, no. 2, pp. 265-274, 2011.

[10] S. Rani and F. Parekh, "Predicting Reservoir Water Level Using Artificial Neural Network," Int. J. Innov. Res. Sci. Eng. Technol., vol. 3, no. 7, pp. 14489-14496, 2014.

[11] S. Mokhtar, W. Ishak, and N. Norwawi, "Modelling of Reservoir Water Release Decision Using Neural Network and Temporal Pattern of Reservoir Water Level," in Fifth International Conference on Intelligent Systems, Modelling and Simulation, 2014, pp. 127-130.

[12] I. González-Taboada, B. González-Fonteboa, F. Martínez-Abella, and J. L. Pérez-Ordóñez, "Prediction of the mechanical properties of structural recycled concrete using multivariable regression and genetic programming," Constr. Build. Mater., vol. 106, pp. 480-499, 2016.

[13] M. Krzy, “AN APPLICATION OF FUNCTIONAL MULTIVARIATE REGRESSION,” vol. 18, no. 3, pp. 433442, 2017.

[14] A. Cancelliere, G. Giuliano, A. Ancarani, and G. Rossi, "Derivation of operation rules for an irrigation water supply by multiple linear regression and neural networks," in Tools for Drought Mitigation in Mediterranean Regions, Vol. 44., Giuseppe Rossi, Antonino Cancelliere, Luis S. Pereira, Theib Oweis, Muhammad Shatanawi, and Abdelaziz Zairi, Eds. Netherland: Springer Netherlands, 2003, pp. 275-291.

[15] A. M. Ticlavilca and M. McKee, "Multivariate Bayesian Regression Approach to Forecast Releases from a System of Multiple Reservoirs," Water Resour. Manag., vol. 25, no. 2, pp. 523-543, Sep. 2010.

[16] V. P. Oikonomou, A. Maronidis, G. Liaros, S. Nikolopoulos, and I. Kompatsiaris, "Sparse Bayesian Learning for subject independent classification with application to SSVEP-BCI," Int. IEEE/EMBS Conf. Neural Eng. NER, pp. 600-604, 2017.

[17] N. Basant, S. Gupta, and K. P. Singh, "Predicting human intestinal absorption of diverse chemicals using ensemble learning based QSAR modeling approaches," Comput. Biol. Chem., 2016.

[18] A. Esmali, K. G. Balderlou, M. Alizadeh, N. Zeinali, and R. H. Gholizadeh, "Prediction of coping styles and happiness based on the maladaptive schema in clients of Aid committee in Urmia Iran.pdf," Res. J. Appl. Sci., vol. 11, no. 4, pp. 144-153, 2016.

[19] P. S. Patki, V. West, and V. V Kelkar, "Classification using Different Normalization Techniques in Support Vector Machine," pp. 4-6, 2013.

[20] Y. Li and J. Ruiz-castillo, "The comparison of normalization procedures based on different classification systems," J. Informetr., vol. 7, no. 4, pp. 945-958, 2013.

[21] T. Jayalakshmi and A. Santhakumaran, "Statistical Normalization and Back Propagation for Classification," vol. 3, no. 1, pp. 1-5, 2011.

[22] M. Eftekhary, P. Gholami, S. Safari, and M. Shojaee, "Ranking Normalization Methods for Improving the Accuracy of SVM Algorithm by DEA Method," vol. 6, no. 10, 2012.

[23] H. Han and K. Men, "How does normalization impact RNA-seq disease diagnosis ?," J. Biomed. Inform., vol. 85, no. November 2017, pp. 80-92, 2018.

[24] K. R. Ku-Mahamud, N. Zakaria, N. Katuk, and M. Shbier, "Flood Pattern Detection Using Sliding Window Technique," in 2009 Third Asia International Conference on Modelling \& Simulation, 2009, pp. 45-50.

[25] K. Ruhana Ku-Mahamud and K. Jia Yun, "Forest Fire Pattern Extraction and Rule Generation using Sliding Window Technique."

[26] S. A. Mokhtar, W. H. W. Ishak, and N. M. Norwawi, "Modelling of reservoir water release decision using neural network and temporal pattern of reservoir water level," Proc. - Int. Conf. Intell. Syst. Model. Simulation, ISMS, vol. 2015-Septe, pp. 127-130, 2015.

[27] W. H. Ishak, K. R. Ku-Mahamud, and N. Norwawi, "Conceptual Model of Intelligent Decision Support System Based on Naturalistic Decision Theory for Reservoir Operation during Emergency Situation," Int. J. Civ. Environ. Eng., vol. 11(2), no. April, pp. 6-11, 2011.

[28] M. H. Hassin, N. Norwawi, and A. Ab-Aziz, "Temporal Case-Based Reasoning for reservoir spillway gate operation recommendation," 2006 Int. Conf. Comput. Informatics, pp. 1-4, Jun. 2006.

[29] W. H. Wan Ishak, K. R. Ku-Mahamud, and N. Md Norwawi, "Modelling of human expert decision making in reservoir operation," J. Teknol., vol. 77, no. 22, pp. 1-5, 2015.

[30] N. Norwawi, "Computational Recognition-Primed Decision Model Based On Temporal Data Mining Approach in A Multiagent Environment For Reservoir Flood Control Decision," 2004.

[31] S. Mokhtar, W. Ishak, and N. Norwawi, "Investigating the Spatial Relationship between the upstream gauging stations and the reservoir," J. Adv. Manag. Sci., vol. 4, no. 6, pp. 503-506, 2016.

[32] K. Ruhana Ku-Mahamud, N. Zakaria, N. Katuk, and M. Shbier, "Flood Pattern Detection Using Sliding Window Technique."

[33] K. R. Ku-Mahamud, N. Zakaria, N. Katuk, and M. Shbier, "Flood Predictive Model generated by temporal data mining technique.".

[34] N. Norwawi, K. R. Ku-Mahamud, and S. Deris, "Recognition Decision-Making Model Using Temporal Data Mining Technique," J. ICT, vol. 4, pp. 37-56, 2005.

[35] M. H. Hassin, N. Norwawi, and A. Ab-Aziz, "Temporal Case-Based Reasoning for reservoir spillway gate operation recommendation," Comput. Informatics, ..., pp. 4-7, 2006. 